\title{
PRODUÇÃO DE FLAVONÓIDES EM MEIOS COMPLEXOS PELO FUNGO Phellinus $S p$.: ESTEQUIOMETRIAS MÍNIMAS E MODELAGEM CINÉTICA E SIMULAÇÃO PARA CULTIVOS EM BATELADA E BATELADA ALIMENTADA
}

\author{
Gustavo Batista ${ }^{1 *}$, Ivan Ilich Kerbauy Veloso ${ }^{1}$, Antonio José Gonçalves Cruz ${ }^{1}$ \\ 1 - Universidade Federal de São Carlos, Programa de Pós-Graduação em Engenharia Química- \\ UFSCar-SP, São Carlos - SP, batistagustavo@live.com
}

\begin{abstract}
Resumo - Este trabalho emprega dados experimentais obtidos por Ma et al. [1] para propor estequiometrias mínimas simplificadas da síntese de flavonóides pelo fungo Phellinus sp. em dois diferentes meios de cultura. Os processos foram simulados através do software AnaBioPlus ${ }^{\circledR}$ para operação nos modos batelada e batelada alimentada. Os resultados mostraram que as equações estequiométricas em fórmulas mínimas foram capazes de predizer o consumo de nutrientes e a formação de produtos de forma satisfatória. A abordagem cinética utilizando as expressões de Contois e LuedekingPiret foi capaz de representar bem os dados experimentais, mostrando-se uma alternativa ao modelo cinético logístico proposto na literatura.
\end{abstract}

Palavras-chave: Phellinus sp., flavonóides, estequiometria mínima, batelada alimentada, modelagem e simulação.

\section{Introdução}

Flavonóides são um grupo de substâncias polifenólicas naturais com propriedades medicinais anti-inflamatórias, antioxidantes e antitumorais comprovadas. Aplicações diversas envolvendo estas substâncias vem sendo estudadas. Porém, ineficiências na produção, seja esta através de extração em plantas ou rotas sintéticas, limitam atualmente o potencial desta tecnologia. Para o advento da produção em larga escala, são importantes o desenvolvimento e a melhoria na eficiência dos processos metabólicos existentes. Um possível biossintetizador é o fungo Phellinus sp. [1].

No trabalho de Ma et al.[1], três meios de cultura foram comparados em biorreator de bancada com volume útil de 5 litros: o meio A, composto por $20,0 \mathrm{~g} / \mathrm{L}$ de glicose, 0,6 g/L de $\mathrm{MgSO}_{4}, 1,0 \mathrm{~g} / \mathrm{L}$ de $\mathrm{KH}_{2} \mathrm{PO}_{4}$, e $4 \mathrm{~g} / \mathrm{L}$ de peptona; o meio B, com a mesma composição do anterior e suplementado por $0,02 \mathrm{~g} / \mathrm{L}$ de ácido naftalenoacético (NAA); e o meio C, com a mesma composição do meio A e adição de $0,03 \mathrm{~g} / \mathrm{L}$ de cumarina. Nestes meios, o cultivo do fungo Phellinus sp. P0988 teve por objetivo a biossíntese de flavonóides, sendo a concentração deste produto empregada para comparação dos três meios de cultura propostos. No presente estudo, serão utilizados os dados experimentais obtidos para os meios de cultura A e B para proposição de estequiometrias mínimas de reação e simulação de cultivos nos modos de operação em batelada e batelada alimentada.

\section{Metodologia}

Determinação da Estequiometria Mínima de Reação

Para representar as reações bioquímicas apresentadas por Ma et al. [1] nos meios A e B, ou seja, os meios de cultura inicial e suplementado com NAA, foram propostas, 
respectivamente, as Equações estequiométricas em fórmulas mínimas 1 e 2, baseadas nas seguintes simplificações:

a) $\mathrm{Na}$ falta de informações mais claras sobre a composição das peptonas utilizadas nos meios de cultura, foi considerada a fórmula molecular da glicina $\left(\mathrm{C}_{2} \mathrm{H}_{5} \mathrm{NO}_{2}\right)$ como representante deste composto (conforme composição de peptona comercial 82962 disponível no catálogo do fornecedor SIGMA-ALDRICH);

b) Os compostos $\mathrm{MgSO}_{4} \mathrm{e} \mathrm{KH}_{2} \mathrm{PO}_{4}$ foram desconsiderados por apresentarem-se em baixas concentrações nos meios de cultura, de modo que toda a reação bioquímica foi representada através dos compostos de carbono, hidrogênio, oxigênio e nitrogênio;

c) A representação do fungo Phellinus sp. se deu por meio da fórmula mínima $\mathrm{CH}_{1,81} \mathrm{O}_{0,52} \mathrm{~N}_{0,21}$ (SHULER);

d) Dentre os flavonóides sintetizados pelo fungo Phellinus sp. foi selecionada a rutina $\left(\mathrm{C}_{27} \mathrm{H}_{30} \mathrm{O}_{16}\right)$ para representação destes compostos.

$$
\mathrm{CH}_{2} \mathrm{O}+\mathbf{a ~ C H} \mathrm{CH}_{5 / 2} \mathrm{~N}_{0,5} \mathrm{O}+\mathbf{b} \mathrm{O}_{2}-->\mathbf{c} \mathrm{CH}_{1,81} \mathrm{O}_{0,52} \mathrm{~N}_{0,21}+\mathbf{d ~ C O} \mathrm{CO}_{2}+\mathbf{e ~} \mathrm{H}_{2} \mathrm{O}+\mathbf{f} \mathrm{CH}_{10 / 9} \mathrm{O}_{16 / 27}
$$

$$
\begin{gathered}
\mathrm{CH}_{2} \mathrm{O}+\mathbf{r C H} \mathrm{CH}_{5 / 2} \mathrm{~N}_{0,5} \mathrm{O}+\mathbf{s ~} \mathrm{O}_{2}+\mathbf{t ~} \mathrm{CH}_{5 / 6} \mathrm{O}_{1 / 6}-->\mathbf{u ~ C H} \mathrm{CH}_{1,81} \mathrm{O}_{0,52} \mathrm{~N}_{0,21}+\mathbf{x ~ C O}+\mathbf{y ~ H} \mathrm{CO}_{2} \mathrm{O}+\mathbf{z} \\
\mathrm{CH}_{10 / 9} \mathrm{O}_{16 / 27}
\end{gathered}
$$

Um balanço dos elementos de reação $\mathrm{C}, \mathrm{H}, \mathrm{O}$ e $\mathrm{N}$ entre reagentes e produtos pode ser aplicado para as Eq. 1 e 2. No caso do meio de cultivo A, por exemplo, os balanços são dados pelas Eqs. 3, 4, 5 e 6, respectivamente:

$$
\begin{gathered}
1+\mathrm{a}=\mathrm{c}+\mathrm{d}+\mathrm{f} \\
2+2,5 * \mathrm{a}=1,81 * \mathrm{c}+2 * \mathrm{e}+10 * \mathrm{f} / 9 \\
1+\mathrm{a}+2 * \mathrm{~b}=0,52 * \mathrm{c}+2 * \mathrm{~d}+\mathrm{e}+16 * \mathrm{f} / 27 \\
0,5 * \mathrm{a}=0,21 * \mathrm{c}
\end{gathered}
$$

Considerando os estados de oxidação intrínsecos dos elementos químicos constituintes das biorreações em estudo (Tabela 1), é possível obter uma equação de balanço adicional para cada meio de cultura avaliado (a Eq. 7 representa o balanço de estados de oxidação entre reagentes e produtos para o meio de cultura A).

$$
4+3 * \mathrm{a}-4 * \mathrm{~b}=4,14 * \mathrm{c}+3,926 * \mathrm{f}
$$

Além disso, é conveniente lançar mão das relações de rendimento estequiométrico $\mathrm{Y}_{\mathrm{X} / \mathrm{S}}$ e $\mathrm{Y}_{\mathrm{P} / \mathrm{S}}$ nos equacionamentos, uma vez que, simplificando as definições matemáticas para reatores que operam em batelada, e considerando as massas fúngica e de flavonóides iniciais iguais a zero [2], estes valores são numericamente iguais a determinados coeficientes do balanço estequiométrico. No caso do balanço equacional em fórmulas mínimas para o meio de cultura A, são simplificadas as relações nas Eqs. 8 e 9. 
Tabela 1 - Somatório dos estados de oxidação dos compostos e moléculas envolvidos nas estequiometrias das Eq. 1 e 2

\begin{tabular}{|c|c|}
\hline $\begin{array}{c}\text { Fórmula mínima } \\
\text { do comp./moléc. }\end{array}$ & $\begin{array}{c}\text { Somatório dos } \\
\text { est. de oxidação }\end{array}$ \\
\hline $\mathrm{CH}_{2} \mathrm{O}$ & 4 \\
\hline $\mathrm{CH}_{5 / 2} \mathrm{~N}_{0,5} \mathrm{O}$ & 3 \\
\hline $\mathrm{O}_{2}$ & -4 \\
\hline $\mathrm{CH}_{1,81} \mathrm{O}_{0,52} \mathrm{~N}_{0,21}$ & 4,14 \\
\hline $\mathrm{CO}_{2}$ & 0 \\
\hline $\mathrm{H}_{2} \mathrm{O}$ & 0 \\
\hline $\mathrm{CH}_{10 / 9} \mathrm{O}_{16 / 27}$ & 3,926 \\
\hline $\mathrm{CH}_{5 / 6} \mathrm{O}_{1 / 6}$ & 4,50 \\
\hline $\mathrm{CH}_{2 / 3} \mathrm{O}_{2 / 9}$ & 4,22 \\
\hline
\end{tabular}

$$
\begin{aligned}
& \mathrm{Y}_{\mathrm{X} / \mathrm{S}}=\frac{\mathrm{r}_{\mathrm{X}}}{\mathrm{r}_{\mathrm{S}}}=\frac{\Delta C_{X}}{-\Delta C_{S}}=\frac{c}{1}=\boldsymbol{c} \\
& \mathrm{Y}_{\mathrm{P} / \mathrm{S}}=\frac{\mathrm{r}_{\mathrm{P}}}{\mathrm{r}_{\mathrm{S}}}=\frac{\Delta C_{P}}{-\Delta C_{S}}=\frac{\boldsymbol{f}}{1}=\boldsymbol{f}
\end{aligned}
$$

Os coeficientes $\mathrm{Y}_{\mathrm{X} / \mathrm{S}}$ e $\mathrm{Y}_{\mathrm{P} / \mathrm{S}}$ para os dois meios de cultivo foram obtidos, respectivamente, por meio do coeficiente angular das retas geradas nos gráficos de $\Delta C_{X}$ versus $-\Delta C_{S}$ e de $\Delta C_{P}$ versus $-\Delta C_{S}$, a partir dos valores extraídos do artigo de Ma et al. [1].

Modelagem e Simulação da Produção de Flavonóides em Batelada e em Batelada Alimentada

Ma et al. [1] utilizaram um modelo logístico (Eq. 10), com cinética independente do substrato, para caracterizar o crescimento da concentração celular $C x$ nos diferentes meios de cultivo dos experimentos operados em batelada. As cinéticas de formação de produto e de consumo de substrato foram baseadas na Equação de Luedeking-Piret (Eq. 11).

$$
\begin{gathered}
\mu=\mu_{\text {máx }} *\left(1-\frac{C_{x}}{C_{x m a ́ x}}\right) \\
\mu_{p}=\alpha * \mu+\beta
\end{gathered}
$$

Este trabalho propõe a utilização do modelo cinético de Contois (Eq. 12) para a representação da cinética de crescimento celular. Tal modelo leva em conta as influências das concentrações de substrato e de células na velocidade específica de crescimento considerando os parâmetros cinéticos $\mu$ máx e Ksx. Uma linearização possível para obtenção simplificada destes parâmetros está apresentada na Eq. 13.

$$
\begin{gathered}
\mu=\frac{\mu_{m a ́ x} * C_{S}}{c_{S}+K s_{x} * C_{x}} \\
\frac{1}{\mu}=\frac{1}{\mu_{\text {máx }}}+\frac{K s_{x}}{\mu_{\text {máx }}} * \frac{C_{x}}{C_{s}}
\end{gathered}
$$


A modelagem dos meios de cultura A e B, para cultivos realizados no modo batelada a volume constante, é expressa pelo sistema de equações diferenciais ordinárias das Eqs. 14, 15 e 16. Estas representam, respectivamente, os balanços de massa para células, produtos (empregando a Eq. 11) e substrato (considerando manutenção celular desprezível), utilizando o modelo cinético de Contois (Eq. 12).

$$
\begin{gathered}
\frac{d C_{x}}{d t}=\mu * C_{x} \\
\frac{d C_{p}}{d t}=(\alpha * \mu+\beta) * C_{x} \\
\frac{d C_{s}}{d t}=-\frac{\mu C_{x}}{Y_{x / s}}-\frac{(\alpha * \mu+\beta) * C_{x}}{Y_{p / s}}
\end{gathered}
$$

A modelagem matemática do processo operado no modo batelada alimentada, com vazão de alimentação constante F para produção de flavonóides por Phellinus sp., será proposta neste trabalho. Nesse modo de operação é possível manipular a concentração de substrato $C s$ e assim a velocidade específica de crescimento $\mu$, possibilitando a obtenção de elevados valores de $C x$ e de $C p$, e também há redução dos riscos de contaminação e mutação inerentes ao processo. Na batelada alimentada, há variação do volume (conforme Eq. 17) após um período inicial de operação em batelada comum. As Eq. 18, 19 e 20 representam, respectivamente, os balanços materiais para a massa de células, de produtos (empregando a Eq. 11) e de substrato (considerando manutenção celular desprezível). Nas equações descritas, $D$ é a taxa de diluição da batelada alimentada, definida como $\mathrm{F} / \mathrm{V}$, e $C s_{F}$ é a concentração de substrato na corrente de alimentação.

$$
\begin{gathered}
\frac{d V}{d t}=F \\
\frac{d C_{x}}{d t}=C_{x} *(\mu-D) \\
\frac{d C_{p}}{d t}=(\alpha * \mu+\beta) * C_{x}-D * C_{p} \\
\frac{d C_{s}}{d t}=D *\left(C s_{F}-C_{s}\right)-\frac{\mu * C_{x}}{Y_{x / s}}-\frac{(\alpha * \mu+\beta) * C_{x}}{Y_{p / s}}
\end{gathered}
$$

Os valores das produtividades globais em células e em produto estão expressas, respectivamente, pelas Eq. 21 e 22. Nestas equações, $\mathrm{Cx}_{0}$ indica a concentração de células no início do cultivo, e tocioso indica o somatório dos tempos de descarga, lavagem, esterilização e carga do reator, fixado em $3 \mathrm{~h}$ para todos os casos. O tempo de cultivo foi considerado o mesmo dos experimentos realizados por Ma et al. [1], sendo para batelada $216 \mathrm{~h}$ e para o cultivo em batelada alimentada o tempo total de $432 \mathrm{~h}$, com $216 \mathrm{~h}$ iniciais de batelada simples. As simulações foram realizadas no software AnaBioPlus $^{\circledR}[3]$.

$$
\begin{aligned}
P_{r x}^{G} & =\frac{C_{x}-C_{x 0}}{t_{\text {OCIOSO }}+t_{\text {CULTIVO }}} \\
P_{r p}^{G} & =\frac{c_{p}}{t_{\text {ocIOSO }}+t_{\text {CULTIVO }}}
\end{aligned}
$$




\section{Resultados e Discussão}

\section{Determinação da Estequiometria Mínima de Reação}

A Tabela 2 apresenta os valores obtidos e os respectivos coeficientes múltiplos de determinação para $\mathrm{Y}_{\mathrm{X} / \mathrm{S}}$. Os valores de $\mathrm{Y}_{\mathrm{P} / \mathrm{S}}$, por sua vez, foram obtidos através do ponto experimental com maior concentração de flavonóides formados. Tal como Ma et al.[1] havia proposto, a adição de NAA ao meio é capaz de aumentar substancialmente a produção de flavonóides por unidade de glicose consumida, sendo um aumento proporcionalmente superior ao apresentado para o crescimento celular.

Tabela 2 - Coeficientes de rendimento calculados $\mathrm{Y}_{\mathrm{X} / \mathrm{S}}$ e $\mathrm{Y}_{\mathrm{P} / \mathrm{S}}$ nas bases mássica e molar

\begin{tabular}{|c|c|c|c|c|c|}
\hline $\begin{array}{c}\text { Meio de } \\
\text { cultura }\end{array}$ & $\begin{array}{c}\mathbf{Y} \mathbf{X} / \mathbf{S} \\
(\mathbf{g X} / \mathbf{g S})\end{array}$ & $\begin{array}{c}\mathbf{Y} \mathbf{X} / \mathbf{S} \\
(\mathbf{C}-\mathbf{m o l X} / \mathbf{C}-\mathbf{m o l S})\end{array}$ & $\mathbf{R}^{\mathbf{2}}$ & $\begin{array}{c}\mathbf{Y P} / \mathbf{S} \\
(\mathbf{g P} / \mathbf{g S})\end{array}$ & $\begin{array}{c}\mathbf{Y} \mathbf{P} / \mathbf{S} \\
(\mathbf{C}-\mathbf{m o l P} / \mathbf{C}-\mathbf{m o l S})\end{array}$ \\
\hline A (inicial) & 0,322 & 0,385 & 0,995 & 0,121 & 0,160 \\
\hline $\begin{array}{c}\text { B (suplem. } \\
\text { com NAA) }\end{array}$ & 0,341 & 0,408 & 0,979 & 0,198 & 0,263 \\
\hline
\end{tabular}

Dispondo dos valores de dois parâmetros de forma direta (Eq. 8 e 9) e resolvendo o sistema de equações estequiométricas em fórmulas mínimas para cada um dos meios de cultura, é possível a obtenção dos coeficientes estequiométricos das Eq. 1 e 2. Os valores estão ilustrados na Tabela 3:

Tabela 3 - Coeficientes estequiométricos obtidos para os meios de cultura

\begin{tabular}{|c|c|c|c|}
\hline \multicolumn{2}{|c|}{ A (Meio Inicial) } & \multicolumn{2}{c|}{ B (Meio Suplementado com NAA) } \\
\hline $\begin{array}{c}\text { Coeficiente } \\
\text { estequiométrico }\end{array}$ & Valor obtido & $\begin{array}{c}\text { Coeficiente } \\
\text { estequiométrico }\end{array}$ & Valor obtido \\
\hline $\mathrm{a}$ & 0,162 & $\mathrm{r}$ & 0,171 \\
\hline $\mathrm{b}$ & 0,566 & $\mathrm{~s}$ & 0,470 \\
\hline $\mathrm{c}$ & 0,385 & $\mathrm{t}$ & 0,0195 \\
\hline $\mathrm{d}$ & 0,617 & $\mathrm{u}$ & 0,408 \\
\hline $\mathrm{e}$ & 0,765 & $\mathrm{x}$ & 0,520 \\
\hline $\mathrm{f}$ & 0,160 & $\mathrm{y}$ & 0,707 \\
\hline- & - & $\mathrm{z}$ & 0,263 \\
\hline
\end{tabular}

Os coeficientes estequiométricos apresentados na Tabela 3 foram então confrontados com as composições químicas originais dos meios de cultivo celular e também com as concentrações de flavonóides obtidas em cada caso, sempre considerando a presença de cinzas na biomassa formada $(5,5 \%$ em massa) e que a glicose é o reagente limitante das reações [2]. Verifica-se nas Tabelas 4 e 5 que os valores experimentais e calculados para as concentrações de peptona foram bastante próximos, de modo que se pode afirmar que a glicina representou bem o conteúdo das peptonas utilizadas. Já os desvios observados para a rutina foram maiores, de forma que a composição dos flavonóides produzidos pelo microrganismo Phellinus sp., nas condições de cultivo, provavelmente tenha sido bastante diversificada.

$\mathrm{Na}$ Tabela 5 verifica-se também que a ordem de grandeza para o coeficiente estequiométrico do NAA calculado para o meio de cultivo B apresenta valores não condizentes. Isso se deve possivelmente ao sistema mal-condicionado de equações estequiométricas (proximidade a uma matriz singular, já que o determinante calculado é muito próximo a zero). Neste caso, pequenas variações nos coeficientes da matriz 
estequiométrica de entrada acarretam em grandes variações na solução do sistema, isto é, a matriz de equações tem um elevado número de condicionamento [4].

Tabela 4 - Comparação entre as concentrações calculadas e experimentais para os compostos do meio de cultura A

\begin{tabular}{|c|c|c|c|c|}
\hline Composto & $\begin{array}{c}\text { Coeficiente } \\
\text { Estequiométrico }\end{array}$ & $\begin{array}{c}\text { Equiv. Conc. } \\
\text { (g/L) }\end{array}$ & $\begin{array}{c}\text { Conc. } \\
\text { Exper. (g/L) }\end{array}$ & Desvio (\%) \\
\hline Glicose & 1 & 20,00 & 20 & 0 \\
\hline Glicina (peptona) & 0,162 & 4,04 & 4 & 1,06 \\
\hline Oxigênio & 0,566 & 12,07 & - & - \\
\hline $\begin{array}{c}\text { Phellinus } \\
\text { sp.(fungo) }\end{array}$ & 0,385 & $6,79 *$ & 6,18 & 9,85 \\
\hline $\begin{array}{c}\text { Dióxido de } \\
\text { Carbono }\end{array}$ & 0,617 & 18,09 & - & - \\
\hline Água & 0,765 & 9,18 & - & - \\
\hline Rutina (flavonóide) & 0,160 & 2,41 & 2,09 & 15,29 \\
\hline
\end{tabular}

Tabela 5 - Comparação entre as concentrações calculadas e experimentais para os compostos do meio de cultura B

\begin{tabular}{|c|c|c|c|c|}
\hline Composto & $\begin{array}{c}\text { Coeficiente } \\
\text { Estequiométrico }\end{array}$ & $\begin{array}{c}\text { Equiv. Conc. } \\
\text { (g/L) }\end{array}$ & $\begin{array}{c}\text { Conc. } \\
\text { Exper. (g/L) }\end{array}$ & Desvio (\%) \\
\hline Glicose & 1 & 20,00 & 20 & 0 \\
\hline Glicina (peptona) & 0,171 & 4,28 & 4 & 6,88 \\
\hline Oxigênio & 0,47 & 10,03 & - & - \\
\hline $\begin{array}{c}\text { Ácido } \\
\text { Naftalenoacético }\end{array}$ & 0,0195 & 0,202 & 0,02 & $\begin{array}{c}\text { Ordem } \\
\text { Grandeza }\end{array}$ \\
\hline $\begin{array}{c}\text { Phellinus } \\
\text { sp.(fungo) }\end{array}$ & 0,408 & $7,19 *$ & 6,29 & 14,37 \\
\hline $\begin{array}{c}\text { Dióxido de } \\
\text { Carbono }\end{array}$ & 0,520 & 15,25 & - & - \\
\hline Água & 0,707 & 8,48 & - & - \\
\hline Rutina (flavonóide) & 0,263 & 3,96 & 3,52 & 12,52 \\
\hline
\end{tabular}

* Considerando presença de 5,5\% de cinzas na massa seca

Modelagem e Simulação da Produção de Flavonóides em Batelada e em Batelada Alimentada

Os valores dos parâmetros $\mu$ máx e Ksx da Eq. 13 foram obtidos por regressão linear, empregando os dados experimentais obtidos por Ma et al. [1], para os meios de cultura A e B. Estes valores estão ilustrados na Tabela 6 e são estatisticamente significativos.

Tabela 6 - Valores de $K s_{x}$ e $\mu_{\text {máx }}$ obtidos por regressão linear do modelo de Contois

\begin{tabular}{|c|c|c|c|c|c|c|c|}
\hline & $\mathbf{K s}_{\mathbf{x}}$ (g/l) & $\begin{array}{c}\text { Erro } \\
\mathbf{K} \mathbf{s}_{\mathbf{x}} \\
(\mathbf{c o n f} \\
\mathbf{9 5 \%}\end{array}$ & $\begin{array}{c}\boldsymbol{\mu}_{\text {máx }} \\
\left(\mathbf{h}^{-\mathbf{1}}\right)\end{array}$ & $\begin{array}{c}\text { Erro } \boldsymbol{\mu}_{\mathbf{m}} \\
\text { áx } \\
(\mathbf{c o n f} \\
\mathbf{9 5 \%}\end{array}$ & $\mathbf{R}^{\mathbf{2}}$ & $\begin{array}{c}\mathbf{F} \\
\text { ANOVA }\end{array}$ & $\begin{array}{c}\mathbf{F} \text { de } \\
\text { signif. } \\
\text { ANOVA }\end{array}$ \\
\hline A (inicial) & 6,54 & 1,84 & 0,0485 & 0,0265 & 0,960 & 97,02 & $6^{*} 10^{-4}$ \\
\hline B (supl. NAA) & 4,46 & 0,34 & 0,0296 & 0,0088 & 0,997 & 1346,66 & $3,29 * 10^{-6}$ \\
\hline
\end{tabular}


Os dados da Tabela 6 foram utilizados nas simulações realizadas no software AnaBioPlus ${ }^{\circledR}$. A formação de flavonóides foi modelada por meio da Eq. 11 para os dois meios de cultura, e foi possível verificar que a formação deste produto é parcialmente associada ao crescimento celular ( $\alpha$ e $\beta$ significativos), sendo os resultados apresentados na Tabela 7.

Tabela 7 - Parâmetros da Equação de Luedeking-Piret obtidos para os diferentes casos avaliados no AnaBioPlus ${ }^{\circledR}$

\begin{tabular}{|c|c|c|}
\hline Meio de cultura & $\boldsymbol{\alpha}$ & $\boldsymbol{\beta}$ \\
\hline A (meio original) & 0,028 & 0,0045 \\
\hline B (suplem. NAA) & 0,0616 & 0,0070 \\
\hline
\end{tabular}

A simulação da operação no modo batelada, empregando o modelo cinético de Contois e os valores dos parâmetros obtidos pela equação de Luedeking-Piret, é apresentada nas Figs. 1 e 2 para os meios de cultura A e B.
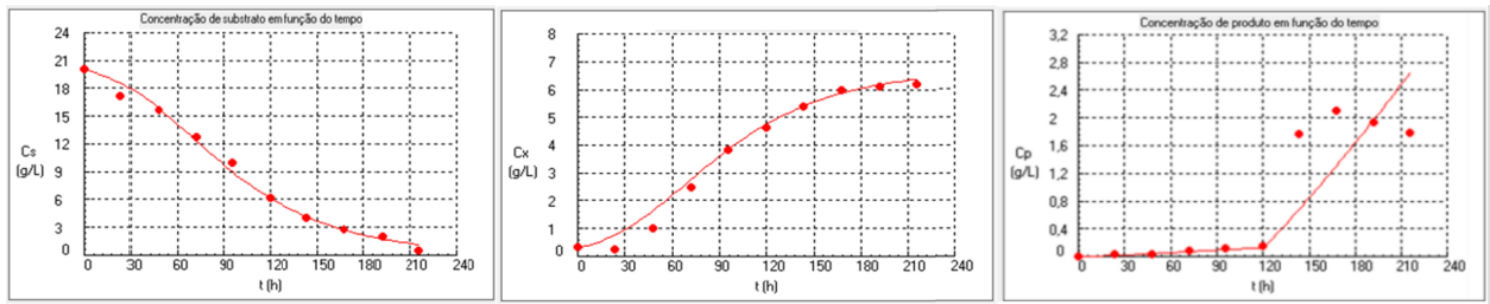

Figura 1 - Concentrações de substrato $(C s)$, de células $(C x)$ e de produto $(C p)$ ao longo do cultivo operado em batelada para o meio de cultura A
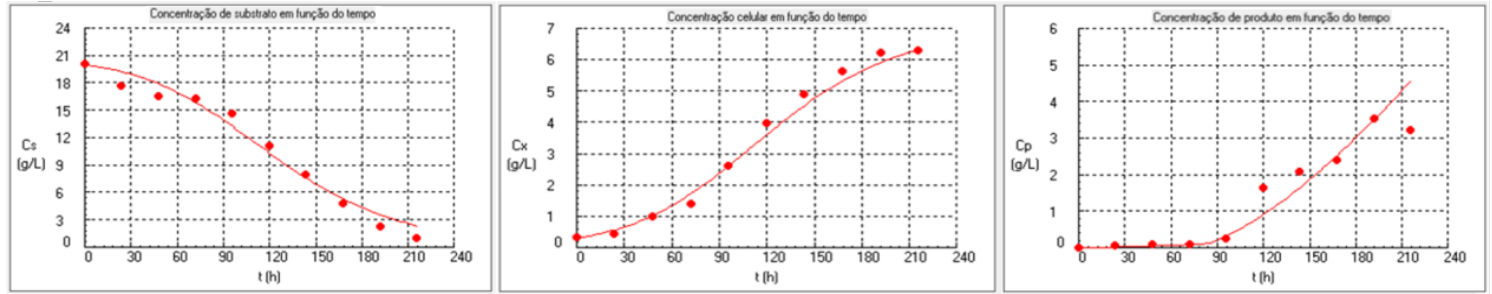

Figura 2 - Concentrações de substrato $(C s)$, de células $(C x)$ e de produto $(C p)$ ao longo do cultivo operado em batelada para o meio de cultura $\mathrm{B}$

A simulação do cultivo no modo batelada alimentada para os dois meios de cultura está ilustrada nas Figs 3 e 4. É possível verificar o aumento na concentração de flavonóides produzidos frente à batelada simples, mantendo a concentração de substrato constante (na batelada alimentada o substrato na alimentação é imediatamente consumido). O efeito da diluição faz com que $C x$ decresça após 216 h.
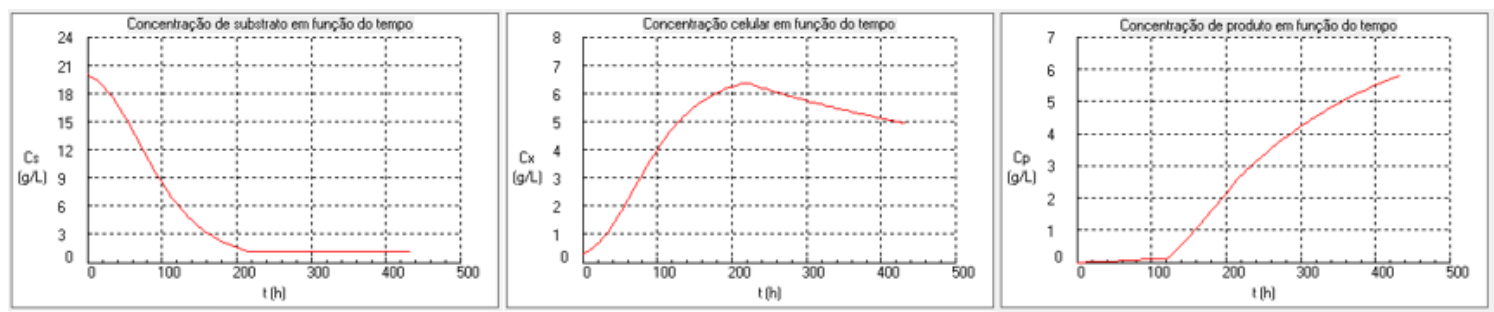

Figura 3 - Concentrações de substrato $(C s)$, de células $(C x)$ e de produto $(C p)$ ao longo do cultivo no modo batelada alimentada com o meio de cultura $A\left(V_{0}=3\right.$ litros, $V_{\text {final }}=$ 5 litros, $\mathrm{D}=0,0024 \mathrm{~h}^{-1}, \mathrm{Cs}_{\mathrm{F}}=10 \mathrm{~g} / \mathrm{l}$ de substrato puro) 

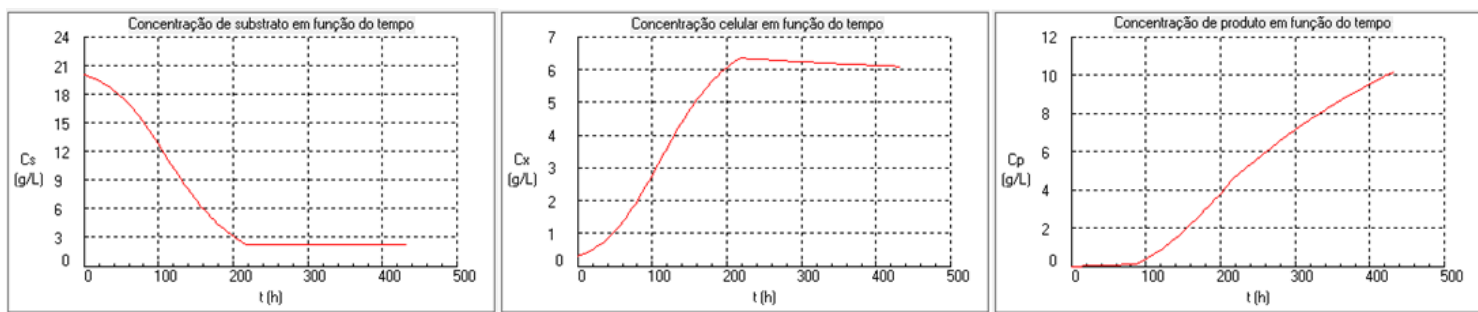

Figura 4 - Concentrações de substrato $(C s)$, de células $(C x)$ e de produto $(C p)$ ao longo do cultivo no modo batelada alimentada com o meio de cultura $\mathrm{B}\left(\mathrm{V}_{0}=3\right.$ litros, $\mathrm{V}_{\text {final }}=$ 5 litros, $\mathrm{D}=0,0024 \mathrm{~h}^{-1}, \mathrm{Cs}_{\mathrm{F}}=19 \mathrm{~g} / \mathrm{l}$ de substrato puro)

Os valores calculados das produtividades em células e em flavonóides, já considerando o somatório dos tempos ociosos, estão apresentados na Tabela 8. Além das produtividades para o meio $\mathrm{B}$ (suplementado por NAA) serem superiores às obtidas para o meio A (como proposto originalmente pelo artigo de Ma et al. [1]), verifica-se que há um aumento de produtividade em produto através da operação em batelada alimentada, quando comparada à operação em batelada simples.

Tabela 8 - Produtividades em células e em produto para diferentes casos avaliados

\begin{tabular}{|c|c|c|c|c|}
\hline & $\begin{array}{c}\text { Batelada / } \\
\text { Meio A }\end{array}$ & $\begin{array}{c}\text { Bat. Alimentada } \\
\text { Meio A }\end{array}$ & $\begin{array}{c}\text { Batelada / } \\
\text { Meio B }\end{array}$ & $\begin{array}{c}\text { Bat. Alimentada } \\
\text { Meio B }\end{array}$ \\
\hline $\begin{array}{c}\text { Prod. células } \\
\left(\mathrm{g} \mathrm{l}^{-1} \mathrm{~h}^{-1}\right)\end{array}$ & 0,028 & 0,011 & 0,028 & 0,013 \\
\hline $\begin{array}{c}\text { Prod. produto } \\
\left(\mathrm{g} \mathrm{l}^{-1} \mathrm{~h}^{-1}\right)\end{array}$ & 0,012 & 0,014 & 0,021 & 0,024 \\
\hline
\end{tabular}

\section{Conclusões}

A determinação dos coeficientes das equações estequiométricas mínimas para a produção de flavonóides, empregando os dados experimentais de Ma et al. [1], teve resultados satisfatórios quando o sistema de equações estequiométricas não se apresentou mal-condicionado. A simulação das operações nos modos batelada e batelada alimentada para dois diferentes meios de cultura e empregando cinéticas de Contois e de Luedeking-Piret mostrou-se plausível para a biossíntese estudada, sendo a produtividade em flavonóides da batelada alimentada um diferencial quando comparada à operação por batelada.

\section{Referências}

[1] MA, X.; PETERSON, E. C.; RUAN, T.; DUAN, X. The influence of naphthaleneacetic acid (NAA) and coumarin on flavonoid production by fungus Phellinus sp.: modeling of production kinetic profiles. Biotechnological Products and Process Engineering, p. 9417-9426, 2015.

[2] SHULER, M. L. KARGI, F. Bioprocess engineering: Basic concepts. Prentice Hall Inc., $2^{\text {a Ed., }} 2002$. 
[3] OLIVEIRA, C. M. JESUS, C. D. F. CENEVIVA, L. V. S. SILVA, F. H. CRUZ, A. J. G. COSTA, C. B. B. BADINO, A. C. AnaBioPlus: a new package for parameter estimation and simulation of bioprocesses. Brazilian Journal of Chemical Engineering, 34: 1065-1082, 2017.

[4] BOLDRINI, J. L. COSTA, S. I. R. FIGUEIREDO, V. L. WETZLER, H. G. Álgebra Linear. Editora Harbra, $3^{\text {a }}$ Ed., 1986. 